\title{
Mildly obscured active galaxies and the cosmic X-ray background
}

\author{
V. Esposito ${ }^{1,2}$ and R. Walter ${ }^{1,2}$ \\ 1 ISDC Data Centre for Astrophysics, University of Geneva, Ch. d'Ecogia 16, 1290 Versoix, Switzerland \\ e-mail: Valentino.Esposito@unige.ch \\ 2 Geneva Observatory, University of Geneva, Ch. des Maillettes 51, 1290 Versoix, Switzerland
}

Received 1 December 2015 / Accepted 12 March 2016

\begin{abstract}
Context. The diffuse cosmic X-ray background (CXB) is the sum of the emission of discrete sources, mostly massive black-holes accreting matter in active galactic nuclei (AGN). The CXB spectrum differs from the integration of the spectra of individual sources, calling for a large population, undetected so far, of strongly obscured Compton-thick AGN. Such objects are predicted by unified models, which attribute most of the AGN diversity to their inclination on the line of sight, and play an important role for the understanding of the growth of black holes in the early Universe.

Aims. The percentage of strongly obscured Compton-thick AGN at low redshift can be derived from the observed CXB spectrum, if we assume AGN spectral templates and luminosity functions.

Methods. We show that high signal-to-noise stacked hard X-ray spectra, derived from more than a billion seconds of effective exposure time with the Swift/BAT instrument, imply that mildly obscured Compton-thin AGN feature a strong reflection and contribute massively to the CXB.

Results. A population of Compton-thick AGN larger than that which is effectively detected is not required to reproduce the CXB spectrum, since no more than $6 \%$ of the CXB flux can be attributed to them. The stronger reflection observed in mildly obscured AGN suggests that the covering factor of the gas and dust surrounding their central engines is a key factor in shaping their appearance. These mildly obscured AGN are easier to study at high redshift than Compton-thick sources are.
\end{abstract}

Key words. galaxies: active - galaxies: Seyfert - diffuse radiation - cosmic background radiation - X-rays: galaxies X-rays: diffuse background

\section{Introduction}

The cosmic X-ray background (CXB) is a broad-band diffuse emission discovered in the early era of X-ray astronomy (Giacconi et al. 1962). We now known that in the X-ray domain, the CXB is the integrated emission of many faint pointlike extragalactic sources, most of which are Seyfert galaxies or quasars.

At energies $<10 \mathrm{keV}$ the $\mathrm{CXB}$ emission has been almost completely resolved into the point-like sources that constitute it (Worsley et al. 2005), thanks to the focusing capabilities of soft $\mathrm{X}$-ray instruments such as XMM-Newton and Chandra. The sum of the fluxes of the sources detected in deep X-ray surveys performed by XMM-Newton in the Lockman Hole (Hasinger et al. 2001) and by Chandra (Giacconi et al. 2002; Alexander et al. 2003) almost reaches the CXB flux level, confirming that the $\mathrm{CXB}$ is the combination of the emission of faint active galactic nuclei (AGN) with different degrees of obscuration.

Current instruments are not sensitive enough to resolve the $\mathrm{CXB}$ at hard X-rays, where the bulk of its emission lies. The extrapolated flux of the AGN population resolved at lower energies is not able to explain the CXB flux at $E \sim 30 \mathrm{keV}$. The strength of X-ray reflection in AGN spectra is a possible solution for this discrepancy. Moreover, the reflection component is the only one that is relevant in heavily obscured, Comptonthick AGN (CTK), that is, sources where the density of the obscuring material is high enough for Compton scattering to dominate $\left(N_{\mathrm{H}}>10^{24} \mathrm{~cm}^{-2}\right)$.
The CXB flux and spectrum have been measured by ASCA/SIS (Gendreau et al. 1995), ROSAT (Miyaji et al. 1998), RXTE/PCA (Revnivtsev et al. 2003), XMM-Newton (De Luca \& Molendi 2004), Chandra (Hickox \& Markevitch 2006) and Swift/XRT (Moretti et al. 2009) at soft X-ray wavelengths, and by HEAO1 (Marshall et al. 1980; Gruber et al. 1999; Revnivtsev et al. 2005) and more recently by Верро-SAX (Frontera et al. 2007), INTEGRAL (Churazov et al. 2007; Türler et al. 2010), and Swift/BAT (Ajello et al. 2008) at hard X-ray wavelengths. Their measurements agree at a level of $\sim 10-15 \%$ throughout the full energy range of the CXB (Revnivtsev et al. 2005; Frontera et al. 2007; Ajello et al. 2008; Moretti et al. 2009).

The percentage of AGN that needs to be CTK to explain the CXB flux is still debated: for example, Gilli et al. (2007) suggest that $50 \%$ of the obscured AGN must be CTK, a similar value is proposed also by Ueda et al. (2014), while Treister et al. (2009) propose a smaller percentage, around $15 \%$.

The method used to perform the CXB synthesis was developed in the seminal works of Setti \& Woltjer (1989) and Comastri et al. (1995) and has been improved in subsequent works. To synthesise the CXB spectrum, three main "ingredients" must be known: an accurate description of the broad band spectra of the various AGN classes, the luminosity function which gives the number density of AGN per comoving volume as a function of luminosity and redshift, and the so-called $N_{\mathrm{H}}$ distribution, that is the distribution of AGN as a function of absorbing column density $\left(N_{\mathrm{H}}\right)$. 
AGN spectra are provided as spectral templates for the various AGN classes: previous works set the parameters of their spectral templates to values representative of observations (e.g. Gilli et al. 2007) or of models (e.g. Ueda et al. 2014). Special care should be used for the CTK template: to model the effect of Compton scattering, especially the scattered component, assumptions on the geometry of the scattering material must be taken, and the spectrum is usually modelled through Monte Carlo simulations assuming a torus geometry for the absorbing material according to the unified AGN model, for example Brightman \& Nandra (2011), Brightman et al. (2014) or the MYTORUS model ${ }^{1}$ proposed by Yaqoob (2012).

The AGN X-ray luminosity function (XLF) is derived from deep surveys (e.g. Ueda et al. 2003, 2014; La Franca et al. 2005; Hasinger et al. 2005; Aird et al. 2010, 2015; Miyaji et al. 2015; Buchner et al. 2015; Ranalli et al. 2016), and so is available only in the soft X-ray range. The $N_{\mathrm{H}}$ distribution can be derived from data (e.g. Gilli et al. 2007, but it is biased against the detection of highly absorbed sources) or from models (e.g. Treister et al. 2009, and references therein).

Since there are many parameters involved in this synthesis, there is a certain level of degeneration in the process, especially in the CTK determination, as shown by Treister et al. (2009) and Akylas et al. (2012). Both authors show that CTK density in the Universe cannot be constrained by the CXB alone, the main reason for which being the lack of a robust CTK spectral template.

In this work we use parameters derived from average spectra of AGN classes measured by Swift/BAT (14-195 keV) to build spectral templates representative of real spectra, resolving the uncertainty in the synthesis process due to the choice of the templates. The shape of the templates strongly affect the synthetised CXB spectrum, especially at hard X-rays where the bulk of the CXB emission lies, and where the contribution of CTK sources is relevant.

The AGN sample and the BAT stacking method are described in detail in Sects. 2 and 3. In Sects. 4 and 5 we describe the ingredients used in the synthesis: spectral templates, XLFs and $N_{\mathrm{H}}$ distributions. In Sect. 6 we present the results, in particular the maximum fraction of CTK allowed by the data and discuss them in Sect. 7.

Throughout this paper we assume $H_{0}=70 \mathrm{~km} \mathrm{~s}^{-1} \mathrm{Mpc}^{-1}$, $\Omega_{\mathrm{M}}=0.3$ and $\Omega_{\Lambda}=0.7$.

\section{The sample}

We used the final sample of 165 sources of Ricci et al. (2011) (R11) to build the stacked spectra. All these sources are Seyfert galaxies detected by INTEGRAL IBIS/ISGRI for which redshift and $N_{\mathrm{H}}$ measurements are available: the redshift spans from 0.001 to 0.162 , the $N_{\mathrm{H}}$ from $4 \times 10^{19}$ to $2.1 \times 10^{24} \mathrm{~cm}^{-2}$.

All these sources have also been detected by Swift/BAT (Tueller et al. 2010; Krimm et al. 2013). Since the BAT field of view is approximately ten times larger than that of ISGRI, stacking BAT data allows unprecedent statistics to be achieved.

Stacked ISGRI spectra of Seyfert galaxies were analysed by R11, introducing a classification based on absorption: Seyfert 2 galaxies are divided into lightly obscured sources (LOB), mildly obscured sources (MOB) and CTK sources.

We notice that the $N_{\mathrm{H}}$ distributions of these four samples are overlapping: there are seven sources classified as Seyfert 1

1 http://mytorus.com
Table 1. Effective exposure and average count rate derived from the mosaic images for each set of stacked images.

\begin{tabular}{lcccc}
\hline \hline Sample & Sources & $\begin{array}{c}\text { Eff. exp. } \\
10^{9} \mathrm{~s}\end{array}$ & Signif. & $\begin{array}{c}\text { Rate } \\
\text { ct s }^{-1} \text { pix }^{-1}\end{array}$ \\
\hline \multicolumn{5}{c}{ Samples based on R11 definition } \\
\hline Seyfert 1 & 44 & 0.627 & 93 & $7.82 \times 10^{-6}$ \\
LOB Seyfert 2 & 34 & 0.478 & 103 & $9.43 \times 10^{-6}$ \\
MOB Seyfert 2 & 27 & 0.384 & 87 & $9.12 \times 10^{-6}$ \\
CTK & 10 & 0.140 & 57 & $10.31 \times 10^{-6}$ \\
\hline \multicolumn{5}{c}{ Samples based on $N_{\mathrm{H}}$} \\
\hline Unabsorbed & 35 & 0.466 & 111 & $8.30 \times 10^{-6}$ \\
LOB1 & 26 & 0.388 & 85 & $7.40 \times 10^{-6}$ \\
LOB2 & 35 & 0.482 & 100 & $6.73 \times 10^{-6}$ \\
MOB & 29 & 0.392 & 91 & $7.13 \times 10^{-6}$ \\
CTK & 10 & 0.140 & 57 & $10.31 \times 10^{-6}$ \\
\hline
\end{tabular}

Notes. For each sample we reported the number of stacked sources, the effective exposure, the significance and the count rate.

with $N_{\mathrm{H}}>10^{22} \mathrm{~cm}^{-2}$, two Seyfert 2 galaxies with $N_{\mathrm{H}}<$ $10^{21} \mathrm{~cm}^{-2}$, and that 29 sources, classified as Seyfert 1.5, were not included in these samples. As a result, we defined an alternate set of samples based exclusively on the $N_{\mathrm{H}}$ : unabsorbed $\left(N_{\mathrm{H}}<10^{21} \mathrm{~cm}^{-2}\right)$, LOB1 $\left(10^{21}<N_{\mathrm{H}}<10^{22} \mathrm{~cm}^{-2}\right)$, LOB2 $\left(10^{22}<N_{\mathrm{H}}<10^{23} \mathrm{~cm}^{-2}\right)$, MOB $\left(10^{23}<N_{\mathrm{H}}<10^{24} \mathrm{~cm}^{-2}\right)$, and $\operatorname{CTK}\left(N_{\mathrm{H}}>10^{24} \mathrm{~cm}^{-2}\right)$.

The total number of sources in each set is reported in the first column of Table 1. These two sets of samples were used to produce stacked images to derive two different sets of spectral templates for the CXB synthesis.

\section{Stacking of BAT data}

The main scientific goal of the Burst Alert Telescope (BAT) on board Swift satellite (Gehrels et al. 2004) is the detection of hard X-ray transients, especially Gamma-Ray Bursts. Thanks to its large field of view (1.4 sr partially-coded) it is also an ideal survey instrument, observing the full sky for very long integrated exposure time.

The Swift/BAT detector consists of $2^{15}$ pixels of CdZnTe (Barthelmy et al. 2005) recording X-ray photons arriving from the sky through a random (50\% open) coded mask, made of 54 000 obscuring lead tiles supported by a honeycomb panel. Each source in the field of view projects a shadow of the mask on the detector plane. The on-board electronic accumulates 80bin spectra from every pixels during a fixed integration period (typically $5 \mathrm{~min}$ ) and send these histograms to ground, together with other data streams. The signal from each source and from the background (dominated by the CXB) can be reconstructed through an image deconvolution. Most sources can only be detected in mosaic of sky images obtained from many ( 1000 s) spacecraft pointings.

The Swift/BAT reduction pipeline for the all-sky survey is described in Tueller et al. (2008, 2010), Baumgartner et al. (2013) and Krimm et al. (2013). Our pipeline follows this closely and is based on the BAT analysis software HEASOFT v 6.13.

A first analysis was performed to derive background detector images. We created sky images (task batsurvey) in the 8 standard energy bands (in keV: 14-20, 20-24, 24-35, 35-50, 50-75, 75-100, 100-150, 150-195) using an input catalogue of 
86 bright sources that have the potential to be detected in single pointings. The detector images were then cleaned by removing the contribution of all detected sources (task batclean) and averaged to obtain one background image per day. The variability of the background detector images was then smoothed pixel-bypixel fitting the daily background values with different function (spline, polynomial). A polynomial model with an order equal to the number of months in the data set adequately represents the background variations. A similar background smoothing function was used by the BAT team.

The BAT image analysis was then run again using these smoothed averaged background maps. The new sky images were then stored in an all-sky pixel database by properly projecting the data on a fixed grid of sky pixel, preserving fluxes (the angular size of the BAT pixels varies in the field of view). This database can then be used to build local images and spectra or lightcurves for any sky position.

The result of our processing was compared to the standard results presented by the Swift team ${ }^{2}$ for individual sources and a very good agreement was found.

To extract the average spectra of the different samples of Seyfert galaxies, we followed the procedure adopted by Walter $\&$ Cabral (2009) and R11. We created $500 \times 500$-pixel mosaic images, modifying the coordinate system of each individual image, and setting the coordinates of each source of the sample to an arbitrarily fixed position $(\alpha=0, \delta=0)$. The geometry of the image was also modified to obtain a uniform point spread function at the centre of the mosaic whatever is the position of the source in the field of view. These mosaic images, built independently for each energy band, provide a stack of all the sources of a given sample.

To minimise the systematics in the mosaic images, we excluded the noisiest images. A total of 201130 sky images were included in the final processing, covering $8 \mathrm{yr}$ from January 2005 to December 2012. Each sky image was in fact included many times, once for each source of the sample present in its field of view. The mosaic images were built with a tangential projection using a factor-of-two oversampling when compared to the individual input sky images. This results in a pixel size of 7.5954 arcmin at the centre of the mosaics. The photometric integrity and accurate astrometry were obtained by calculating the intersection between input and output pixel, and weighting the count rates according to the overlapping area.

The average signal, which was extracted from the mosaic for each individual sample, the exposures and the number of sources used, are reported in Table 1. The detection significances of the stacked sources in the mosaics are calculated as the number of counts divided by the square root of the variance and range between $111 \sigma$ and $57 \sigma$. The effective exposure obtained at the centre of the mosaics are between 0.627 Gs and 0.140 Gs.

The eight-bin-stacked spectrum for each AGN sample was extracted from the mosaic images using mosaic_spec ${ }^{3}$. The standard BAT response matrix depends on the off-axis angle and cannot be used when data from many off-axis angles are averaged. To produce a suitable matrix we followed the same procedure adopted by the BAT team for the survey work (Baumgartner et al. 2013), which consists in tuning the on-axis response to obtain a correct spectrum for the Crab nebula. The tuning accounts for the fact that a smaller fraction of the low energy photons are detected on average over the field of view when compared

\footnotetext{
2 http://swift.gsfc.nasa.gov/results/bs70mon/

3 From the OSA package, http://isdc.unige.ch/integral/ analysis
}

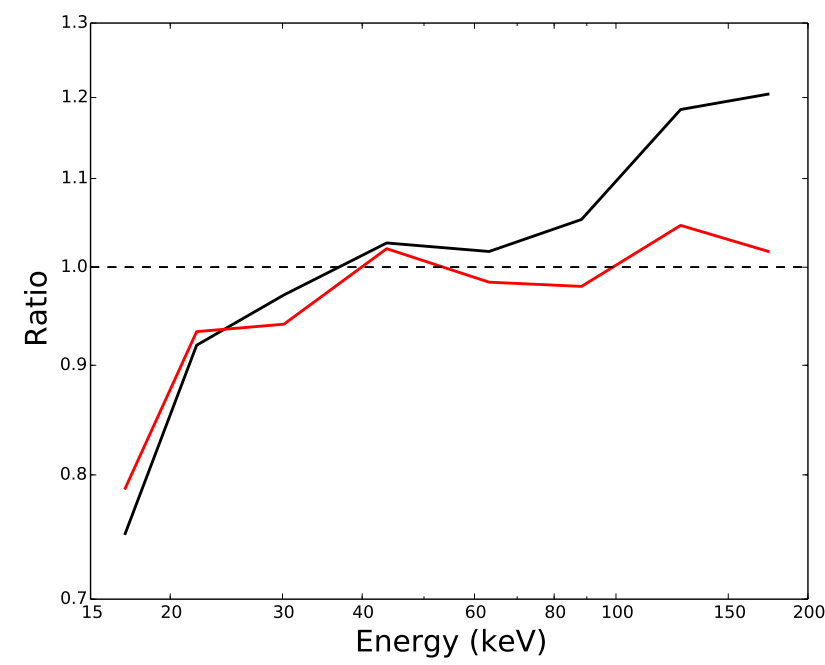

Fig. 1. Ratio between the averaged Crab spectrum and a powerlaw (red: our work; black: from Baumgartner et al. 2013), using an on-axis response.

with the on-axis expectation, largely because of absorption in the honeycomb mask supporting structure. The correction therefore depends on the average spectrum of the Crab nebula. Figure 1 shows the ratio between the observed spectrum (obtained by us and by Baumgartner et al. 2013) and the powerlaw $\left(F_{v} \sim v^{-1.15}\right)$ model representing the Crab nebula, obtained assuming the onaxis response. The effect of the absorption at low energy is clearly observed. Up to $80 \mathrm{keV}$ the observed spectra are consistent within a few \%. At higher energies Baumgartner et al. (2013) required an additional correction that we are not observing. The red curve in Fig. 1 is very similar to the NOMEX absorption model used for the supporting structure of the IBIS mask on board INTEGRAL (Lubinski, priv. comm.). We finally used the response matrix provided by Baumgartner et al. $(2013)^{4}$, modified to obtain a good powerlaw adjustment to the spectrum of the Crab nebula, in particular above $80 \mathrm{keV}$. A systematic uncertainty of $2 \%$ has been added in all spectral model adjustments to account for the uncertainty related to the off-axis effects at low energies. We also built a second response matrix assuming a broken powerlaw for the Crab spectrum (Jourdain et al. 2008) and found that the resulting spectral parameters (Table 2) were identical within the uncertainties.

\section{Stacked spectra and spectral templates}

Stacking the Swift/BAT data as described in Sect. 3 allows us to obtain average spectra for the Seyfert galaxy classes in the BAT energy bandpass (14-195 keV) characterised by different absorption levels. The parameters derived from these spectra are then used as spectral templates in the CXB synthesis.

The stacked spectra of the samples built for $\log \left(N_{\mathrm{H}}\right)<24$ were fitted with the pexrav model modified for photoelectric absorption. The pexrav model consists of an exponentially cutoff powerlaw plus reflection from an infinite slab of neutral material, representing an accretion disk (Magdziarz \& Zdziarski 1995). The absorption was fixed to the central value (in logarithmic space) of the included sources.

The spectral fit has been performed with XSPEC (version 12.8.2) using the $\chi^{2}$ minimisation method. As described in Sect. 3 the spectra are binned in 8 energy bins, and the stacked

4 http://swift.gsfc.nasa.gov/results/bs70mon/ 


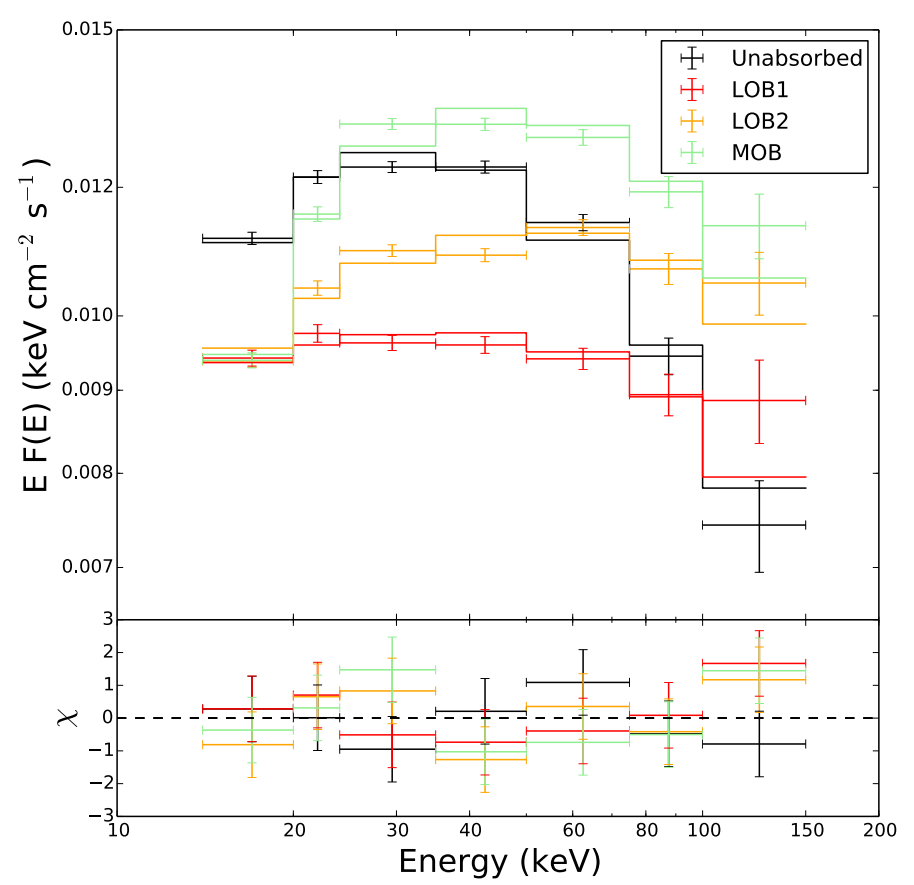

Fig. 2. Swift/BAT stacked spectra of the samples based on $N_{\mathrm{H}}$ and fitted with phabs * pexrav model.

Table 2. Spectral parameters (spectral index $\Gamma$ and reflection $R$ ) derived from the fits of the stacked spectra and used for the spectral templates of the CXB synthesis.

\begin{tabular}{lccccc}
\hline \hline Sample & $\log \left(N_{\mathrm{H}}\right)$ & $\Gamma$ & $R$ & $\Delta \Gamma$ & $\Delta R$ \\
\hline \multicolumn{5}{c}{ Stacked spectra based on R11 definition } \\
\hline Seyfert 1 & 0 & 1.86 & 0.17 & 0.02 & 0.14 \\
LOB Seyfert 2 & 22 & 1.68 & 0.0 & 0.02 & 0.04 \\
MOB Seyfert 2 & 23.5 & 1.74 & 0.88 & 0.02 & 0.12 \\
\hline \multicolumn{6}{c}{ Stacked spectra based on $N_{\mathrm{H}}$} \\
\hline Unabsorbed & 0 & 1.89 & 0.77 & 0.02 & 0.2 \\
LOB1 & 21.5 & 1.82 & 0 & 0.02 & 0.04 \\
LOB2 & 22.5 & 1.72 & 0 & 0.02 & 0.21 \\
MOB & 23.5 & 1.72 & 0.62 & 0.02 & 0.2 \\
\hline
\end{tabular}

Notes. $1 \sigma$ errors derived from the fits are reported in the last two columns. The absorption is always a fixed parameter. pexrav fixed parameters: $E_{\mathrm{c}}=200 \mathrm{keV}, \cos \theta=0.45$, solar abundances.

sources are not detected in the highest one, so it is ignored in the spectral fit. The spectra are shown in Fig. 2 with the best fit model and residuals.

These impressive statistics (the total number of photons in the spectra ranges between $\sim 12000$ and $~ 34000$ ) obtained with the stacking method, allow us to measure, with good accuracy, the relevant spectral parameters, specifically the spectral in$\operatorname{dex} \Gamma$ and the covering factor of the reflecting material $R$. Table 2 reports these parameters for each spectrum. The cutoff energy $E_{\mathrm{c}}$ lies beyond the upper threshold of the BAT energy band and cannot be constrained by the fit, so we fixed it at $E_{\mathrm{c}}=200 \mathrm{keV}$, as it is unlikely to have higher values (Malizia et al. 2014). We fixed also the inclination angle $\theta$ at the value of $\cos \theta=0.45$ and solar abundancies were assumed.

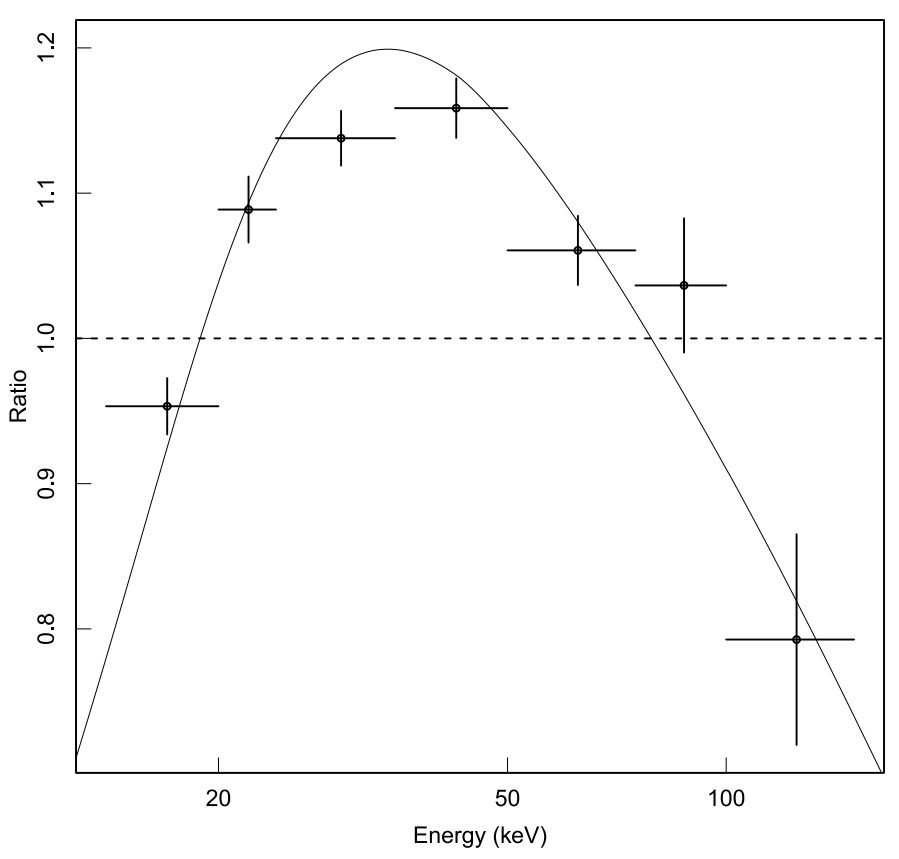

Fig. 3. Ratio of MOB versus LOB1 spectra. The spectra are derived from the samples based on $N_{\mathrm{H}}$ definition. The curve is the ratio between the two best fit models fitted with the pexrav model.

We notice that the spectral index $\Gamma$ becomes softer for absorbed sources, in agreement with the results of Burlon et al. (2011) and Vasudevan et al. (2013), who also analysed Swift/BAT spectra stacked according to absorption. The mildly obscured samples (MOB Seyfert 2 and MOB) feature a strong curvature, a sign of a high reflection levele (Table 2). This reflection amplitude is larger than observed in the spectra derived for lightly absorbed samples and comparable with the reflection shown by the unabsorbed sample. The parameters listed in Table 2 are model dependent and we do not know if e.g. the cutoff energy is the same in unabsorbed or absorbed sources, but the different curvatures are confirmed in Fig. 3, which is also not affected by uncertainties in the instrumental calibration and response, confirming that the difference in curvature observed between the LOB and MOB stacked spectra is not an artifact. This confirms with much higher signal to noise and solidity, previously obtained results (R11; Vasudevan et al. 2013).

This effect is not apparent in Burlon et al. (2011), since they stacked only unabsorbed $\left(N_{\mathrm{H}}<10^{22} \mathrm{~cm}^{-2}\right)$ and absorbed $\left(N_{\mathrm{H}}>\right.$ $10^{22} \mathrm{~cm}^{-2}$ ) spectra. Thus, we conclude that although the stacking method of Burlon et al. (2011) and Vasudevan et al. (2013) are different from the one adopted in this work, the results of all three agree.

The model dependence of the parameters in Table 2 does not matter very much for the CXB synthesis as long as the spectral templates provide a good representation of the average spectra. We have verified that fitting the data with a different fixed cutoff energies provides different parameters but produce finally the same synthesied CXB. This will be discussed in more detail in Sect. 7 .

To generate CTK spectral template, we adopted the MYTORUS model (Yaqoob 2012), which properly calculates the absorption due to Compton scattering. This model assumes a powerlaw emission as primary component and a donut-shaped torus with fixed solar abundancies surrounding the central emitter. It provides a transmitted component and a scattered component, the latter playing the role of the pexrav- reflection component. 


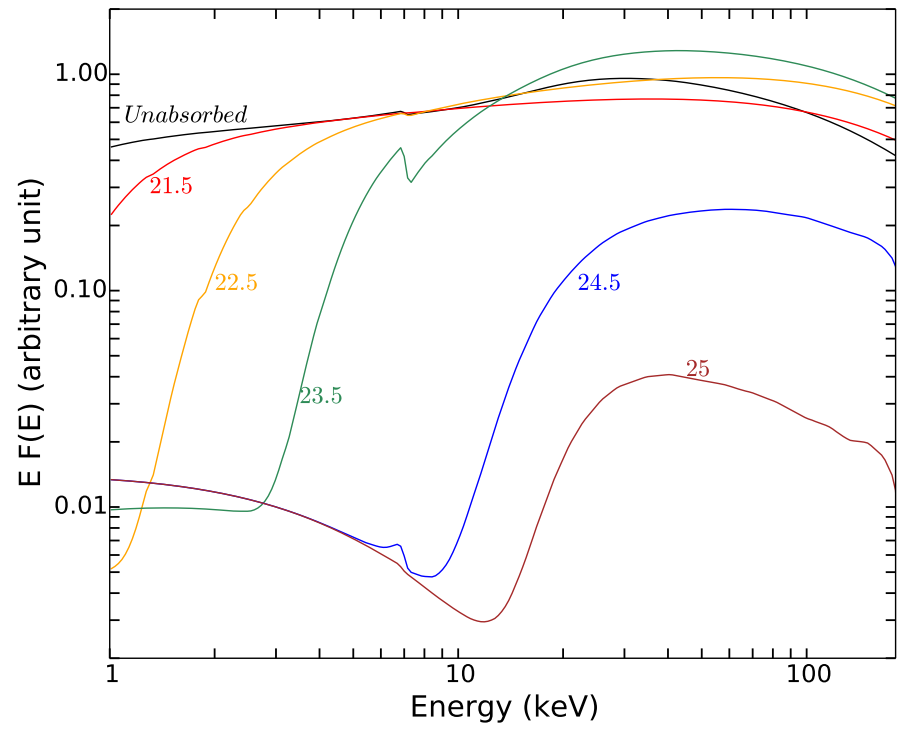

Fig. 4. Spectral templates generated with the parameters presented in Table 2, stacked spectra based on $N_{\mathrm{H}}$. The templates are normalized such that the integrated emission of their primary component in the $2-10 \mathrm{keV}$ band is equal to 1 . The numbers identify the templates by their $\log \left(N_{\mathrm{H}}\right)$ value. The parameters of the CTK templates $\left(\log \left(N_{\mathrm{H}}\right)>\right.$ 24) are given in the text.

The shapes predicted by the pexrav reflected component and the MYTORUS- scattered component are significantly different for $\log \left(N_{\mathrm{H}}\right) \geq 24 \mathrm{~cm}^{-2}$.

The transmitted and scattered components of the MYTORUS model can be fitted separately, in order to measure the relative strength of the scattered component. Since our template needs to represent an average spectrum, we fixed this relative strength to 1 . As a primary component we use a cutoff powerlaw with $\Gamma=1.9$ and $E_{\mathrm{c}}=200 \mathrm{keV}$, and the transmitted component is modelled with a powerlaw truncated at $200 \mathrm{keV}$, with the same spectral index, scattered by the absorbing torus. The inclination angle is fixed at $90^{\circ}$.

We introduced two templates for Compton-thick sources, separating them into moderately obscured $\left(10^{24}<N_{\mathrm{H}}<\right.$ $\left.10^{25} \mathrm{~cm}^{-2}\right)$ and deeply obscured CTK $\left(N_{\mathrm{H}}>10^{25} \mathrm{~cm}^{-2}\right)$, as in previous works (Gilli et al. 2007; Ueda et al. 2014). The parameters used for the two templates are the same except for the absorption.

To conclude the template modelling, we added a scattered component in the range $1-10 \mathrm{keV}$ in obscured spectra (i.e. with $\log \left(N_{\mathrm{H}}\right)>21$ ), following the recipe of Gilli et al. (2007), to model the X-ray emission in excess of the absorbed powerlaw commonly observed in Seyfert 2 galaxies. It is modelled as a cutoff powerlaw with $E_{\mathrm{c}}=5 \mathrm{keV}$, the same spectral index as the main component, and normalisation fixed at $3 \%$ of the normalisation of the main component. Since the iron line and other relevant emission lines are located at energies below the BAT lower energy threshold, we did not include any line in our modelling.

With the spectral parameters, we generated spectral templates in the range $1-500 \mathrm{keV}$ (Fig. 4) which were used for the CXB synthesis. Previous works, for example, Gilli et al. (2007), Ueda et al. (2014), assumed a Gaussian dispersion of spectral indexes. Since our templates are derived from stacked spectra, they represent the average value of the sample, so we have chosen to not include any dispersion.
We should point out that these spectral templates are derived by stacking local AGN. We assume that they are representative of AGN up to $z \sim 1$.

\section{X-ray luminosity function and $N_{H}$ distribution}

The XLF gives the AGN number density per comoving volume as a function of intrinsic unabsorbed luminosity and redshift. We used the XLFs of Ueda et al. (2003), Hasinger et al. (2005), Ueda et al. (2014), and Miyaji et al. (2015; U03, H05, U14, and M15 respectively). U03, U14, and M15 built their XLFs in the 2-10 keV energy band, for both "type-1" and "type-2" objects, and using samples of 247, 4039 and 3200 AGN, respectively. The XLF of H05 were built in the $0.5-2 \mathrm{keV}$ energy band and used 944 AGN "type-1" objects. Gilli et al. (2007; G07) compared the H05 XLF with other XLFs (U03; La Franca et al. 2005), built from "type-1" plus "type-2" objects, in order to estimate a luminosity-dependent ratio of obscured versus unobscured objects $R_{\mathrm{O} / \mathrm{u}}\left(L_{\mathrm{X}}\right)$, and corrected the H05 XLF to obtain the density of obscured AGN. We used the same correction.

The XLFs are derived by fitting the sample with an analytical empirical smoothed two-powerlaw formula which has been found to fit the data well in the local universe. This expression is then corrected for a density evoluction factor, $e_{\mathrm{d}}$, to set the dependence on the redshift. The analytical expression of the density evolution factor is defined according to an evolution model. U03, H05, and U14 agreed that the best fit to the data is achieved using the luminosity dependent density evolution (LDDE) model, which is to say that $e_{\mathrm{d}}$ depends on both the $\mathrm{X}$-ray luminosity $L_{\mathrm{X}}$ and the redshift $z$, rather than other models such as the pure luminosity evolution (PLE) model, where the density evolution factor depends on the redshift $z$ alone. We used the best fit XLF models provided by U03, H05, U14, and M15, which are described as:

$$
\frac{\mathrm{d} \Phi\left(L_{\mathrm{X}}, z\right)}{\mathrm{d} \log L_{\mathrm{X}}}=A\left[\left(\frac{L_{\mathrm{X}}}{L_{*}}\right)^{\gamma_{1}}+\left(\frac{L_{\mathrm{X}}}{L_{*}}\right)^{\gamma_{2}}\right]^{-1} e_{\mathrm{d}}\left(L_{\mathrm{X}}, z\right)
$$

and the density evolution factor:

$e_{\mathrm{d}}\left(L_{\mathrm{X}}, z\right)= \begin{cases}(1+z)^{p_{1}} & \text { if } z \leq z_{\mathrm{c}} \\ \left(1+z_{\mathrm{c}}\right)^{p_{1}}\left[(1+z) /\left(1+z_{\mathrm{c}}\right)\right]^{p_{2}} & \text { if } z>z_{\mathrm{c}}\end{cases}$

with

$z_{\mathrm{c}}\left(L_{\mathrm{X}}\right)= \begin{cases}z_{\mathrm{c}}^{*}\left(L_{\mathrm{X}} / L_{\mathrm{X}, \mathrm{c}}\right)^{\alpha} & \text { if } L_{\mathrm{X}} \leq L_{\mathrm{X}, \mathrm{c}} \\ z_{\mathrm{c}}^{*} & \text { if } L_{\mathrm{X}}>L_{\mathrm{X}, \mathrm{c}} .\end{cases}$

While U03 used exactly these equations, H05 introduced an extra smooth dependence on $L_{\mathrm{X}}$ in Eq. (2), in the indexes $p_{1}$ and $p_{2}$, while U14 introduced an extra variation in the slope at high redshift in Eq. (2), which therefore has three slopes, $p_{1}, p_{2}$, and $p_{3}$, and two redshift thresholds, $z_{\mathrm{c}, 1}^{*}$ and $z_{\mathrm{c}, 2}^{*}$. M15 used the same definition of U14, but fixed different parameters in the fit. The differences between these XLFs are due not only to the dataset used, but also to these small, but probably relevant, different parametrisations of the analytical equations.

In the $\mathrm{CXB}$ synthesis process, the analytic equation of the XLF is multiplied by the luminosity $L_{X}$ and then integrated in the range $42<\log L_{\mathrm{x}}<48$, and $0<z<5$. The uncertainties of the XLFs at high luminosity and high redshift are not relevant for the CXB synthesis as the contribution to the CXB of AGN in this range is negligible.

Figure 5 shows the U14 XLF for two redshift bins with confidence regions at $1 \sigma$ and $2 \sigma$ and the U03 XLF for comparison. 


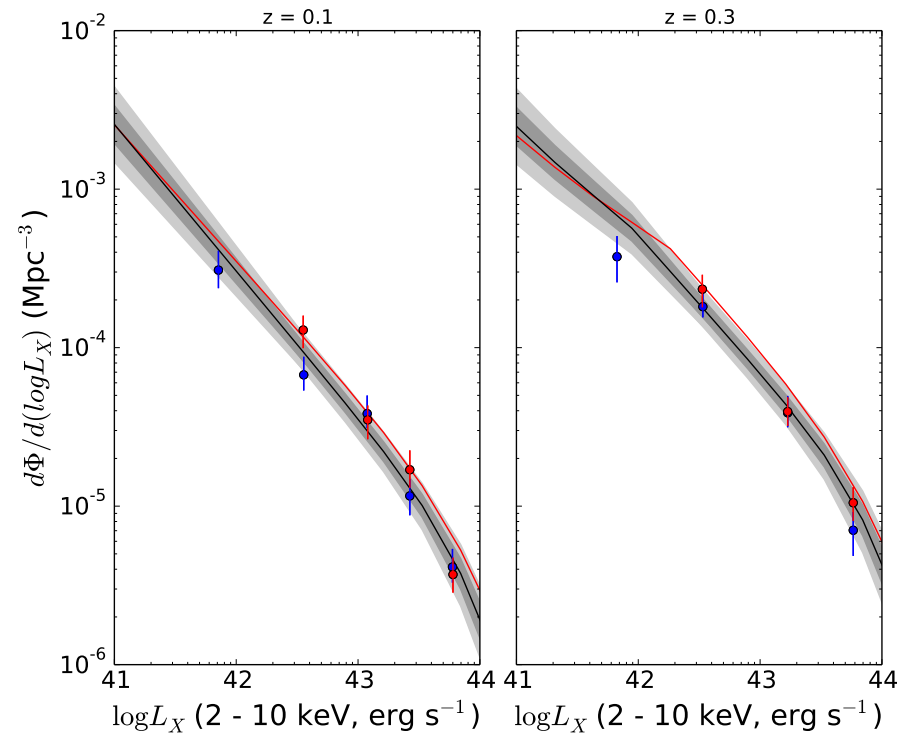

Fig. 5. XLF of U14 plotted at redshift $z=0.1$ and $z=0.3$ in the range $41<\log L_{\mathrm{x}}<44$. The black line represents the best fit, grey regions show the effect of uncertainties in the parameters: dark (light) grey correspond to $1 \sigma(2 \sigma)$ region. The red line is U03 XLF. Red and blue points are taken from U14, and represent the data derived from the soft $(<2 \mathrm{keV})$ and hard $(>2 \mathrm{keV}) \mathrm{X}$-ray bands respectively, converted in 2 to $10 \mathrm{keV}$ luminosities as explained in U14.

The confidence region is mostly based on the uncertainties on $\gamma_{1}$ and $L_{*}$. The uncertainty on the XLF normalisation can be up to approximately $10 \%(1 \sigma)$. The CXB synthesis of U14 (whose XLF is mostly based on Chandra surveys) falls 10-20\% short of the CXB flux measured by Chandra. This indicates that the $\mathrm{XLF}$ is probably underestimated even though other sources can contribute to the CXB in the soft X-ray band.

The percentage of AGN with different $N_{\mathrm{H}}$ must be known to synthetise the CXB. We used the $N_{\mathrm{H}}$ distribution derived by G07 and Treister et al. (2009; T09) and also derived it to obtain the best match between the observed and syntetised CXB. These $N_{\mathrm{H}}$ distributions, displayed in Fig. 7 show significant discrepancies.

It must be pointed out that these $N_{\mathrm{H}}$ distributions do not depend on redshift and luminosity, in contrast to some observations (La Franca et al. 2005; Hasinger 2008; Aird et al. 2010, 2015; Miyaji et al. 2015; Buchner et al. 2015; U14). To account for this effect, U14 propose an empirical analytical function for the $N_{\mathrm{H}}$ distribution dependent on $L_{\mathrm{X}}$ and $z$. We tested that function. together with the U14 and M15 XLFs, who used it in their works.

The CTK AGN are considered separately from the above $N_{\mathrm{H}}$ distributions, their fraction is evaluated by adjusting the CXB synthesis to the observations.

\section{Synthesis of the CXB}

\subsection{Comparison with previous works}

Figure 6 shows different synthetised spectra, obtained by selecting combinations of XLF and $N_{\mathrm{H}}$ distribution used in previous works (G07, T09, U14, and M15) and using the set of templates derived from $S$ wift/BAT. These spectra are plotted against Swift/XRT (Moretti et al. 2009), ASCA SIS (Gendreau et al. 1995), INTEGRAL (Churazov et al. 2007), Swift/BAT (Ajello et al. 2008), HEAO1 (Gruber et al. 1999), ROSAT (Miyaji et al. 1998), and Chandra (Hickox \& Markevitch 2006) data. We do not perform any fit or renormalisation on the data. Figure 6 also shows the separated contributions of unabsorbed, LOB (which, here, is the sum of $\log \left(N_{\mathrm{H}}\right)=21.5$ and $\log \left(N_{\mathrm{H}}\right)=22.5$ ), and MOB AGN. The CTK fraction is usually estimated to fill the gap between the total contribution of Compton-thin objects and the data. The CTK fraction shown corresponds to the one estimated in the paper in question.

The main differences between the synthesis models are caused by the XLF. Even without CTK sources, H05 and U03 XLFs are in good agreement with the data above $30 \mathrm{keV}$. The U14 XLF instead produces a CXB spectrum, 10-20\% fainter than that observed by Chandra, with a wide gap between the data and the spectrum at $\sim 30 \mathrm{keV}$. As mentioned previously, this $\mathrm{XLF}$ is too faint to account for the CXB at soft X-rays, and requires a large percentage of CTK sources to match the CXB at hard X-rays.

\subsection{Fitting the Compton-thick contribution}

The CTK contribution to the CXB is estimated by varying the CTK fraction through a $\chi^{2}$ minimisation. To investigate the effect of using the BAT templates (Sect. 4), the CXB is synthetised with the combinations of XLFs and $N_{\mathrm{H}}$ distributions used by G07, T09, and U14, to estimate the CTK fraction. We used the same CXB datasets as in the papers in question. In Table 3 we report the flux due to the CTK sources at the peak of the integrated spectrum. With the BAT templates, we obtain significantly fewer CTK sources. However, in all these cases, the fit to the CXB is not acceptable. Table 3 refers to results obtained with the BAT spectral templates derived from the samples based on absorption; using the samples based on Seyfert-type leads to similar results.

We then compared the CXB synthesis with the CXB observed with ROSAT, Swift/XRT, Swift/BAT, and INTEGRAL. We considered only the ROSAT data above $1 \mathrm{keV}$ to exclude the soft excess component from the CXB modelling. These datasets, as well as those of XMM-Newton (Moretti et al. 2009) and Chandra, are cross-calibrated well and feature similar CXB normalisation. Their calibration also matches those of the recent XLF obtained with deep soft X-ray surveys by XMM and Chandra.

Table 4 shows the estimates of the CTK fraction for the combinations of XLFs and $N_{\mathrm{H}}$ distributions shown in Fig. 6. We report the flux of the CTK contribution at the peak and the fraction of CTK objects (assuming that all CTK are in the $N_{\mathrm{H}}$ range $10^{24}-10^{25} \mathrm{~cm}^{-2}$ ) needed to produce this flux, and the corresponding $\chi_{\text {red }}^{2}$. With the XLF and $N_{\mathrm{H}}$ distribution used by G07 and T09 and with the BAT templates, the amount of CTK allowed by the synthesis is less than in the papers we refer to, confirming that BAT templates produce higher CXB flux. Even with the XLF and $N_{\mathrm{H}}$ distribution of U14, we obtain a smaller CTK percentage. The fit to the CXB data is not good regardless of combitation of XLF and $N_{\mathrm{H}}$ distribution considered: the BAT templates and the previous models together cannot adequately represent the data.

\subsection{Fitting the $N_{H}$ distribution}

To achieve a better representation of the CXB spectrum, we adjusted the $N_{\mathrm{H}}$ distribution directly to the data. We performed several fits with different assumptions to investigate possible systematics effects: we used the XLF of U14 or U03, we fixed the maximum $N_{\mathrm{H}}$ of the distribution at $10^{25} \mathrm{~cm}^{-2}$, as done by T09, or at $10^{26} \mathrm{~cm}^{-2}$, as in G07 and U14 (the implications of this choice 
V. Esposito and R. Walter: Mildly obscured active galaxies and the cosmic X-ray background

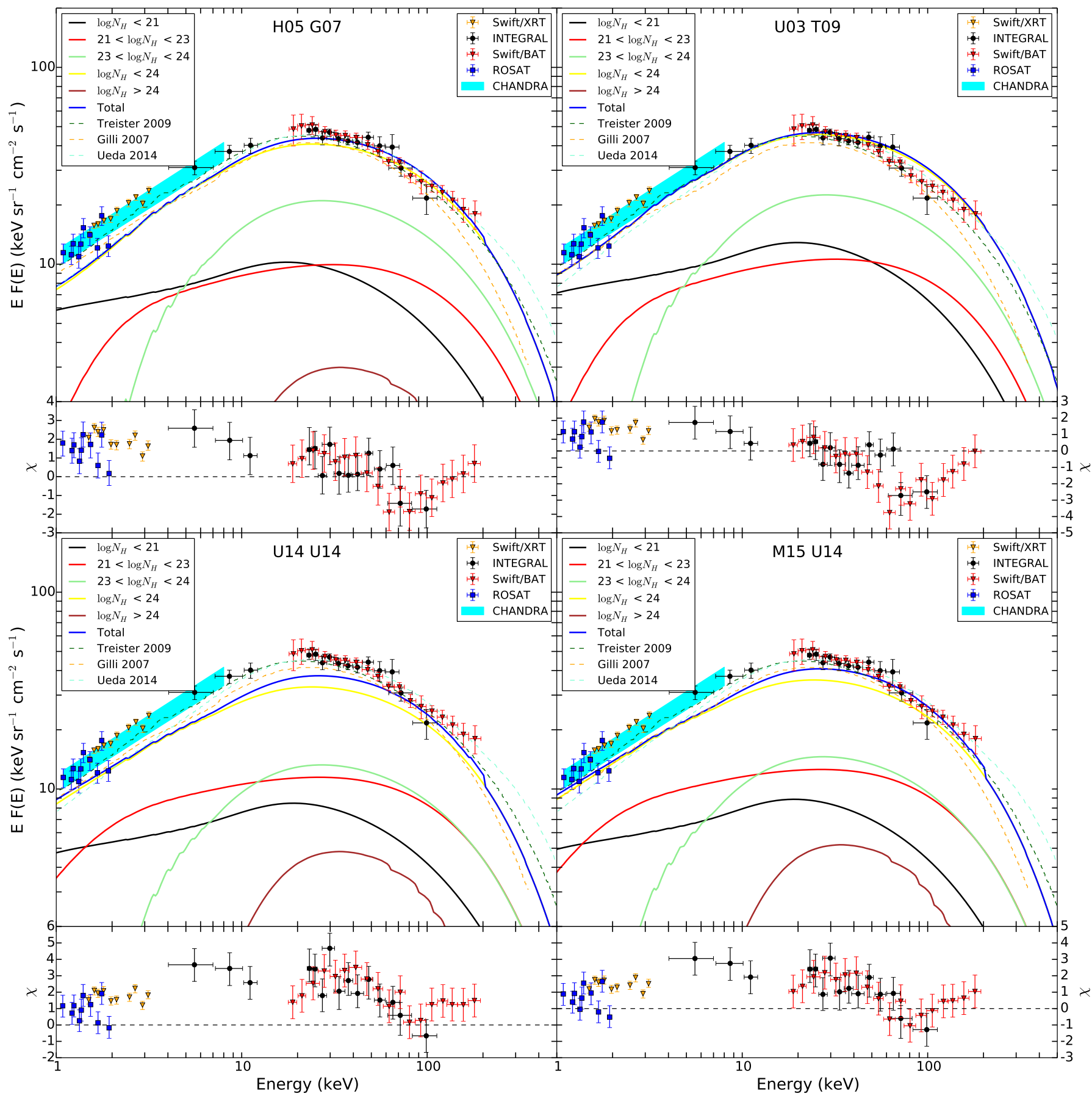

Fig. 6. Synthesis of the CXB (thick blue line), based on BAT spectral templates for different XLFs and $N_{\mathrm{H}}$ distributions. The title of each panel quotes the papers from which the XLFs and $N_{\mathrm{H}}$ distributions are taken. The data from Swift/XRT (orange triangles), ASCA SIS (green squares), INTEGRAL (black points), Swift/BAT (red squares), HEAO1 (grey triangles), ROSAT (blue triangles), and Chandra (cyan region) are shown. Each panel shows the spectra and the difference between the data and the synthesis.

are discussed later), and we used the BAT templates based on samples defined according to $N_{\mathrm{H}}$ or Seyfert type (see Sect. 3).

There are several sources of uncertainty over the CXB normalisation and synthesis. We improved the uncertainty on the spectral templates by using average hard X-ray spectra. The integrated XLF varies by $15 \%$ for different assumptions and by $13 \%$, taking $1 \sigma$ statistical uncertainties of the XLF into account. The CXB normalisation between the various datasets varies by $10-15 \%$ and the CXB synthesis flux produced with the U14 XLF falls 10-20\% below the Chandra CXB data.
In addition, we investigated the fraction of absorbed AGN which still escape detection. We simulated spectra with $N_{\mathrm{H}}$ uniformly distributed into 6 linearly spaced bins in the range $10^{23}-10^{24} \mathrm{~cm}^{-2}$ (corresponding to our MOB sources definition), with the spectral index $\Gamma=1.72$ derived for the MOB sources (Table 2), and convolving them with the Chandra matrix response assuming the total exposure of the Deep Chandra Surveys used by U14. We then looked for the deabsorbed luminosity required to be detected by Chandra, and integrated U14 XLF from to the lower $\operatorname{limit}\left(\log L_{\mathrm{x}}=42\right)$ to this luminosity 
Table 3. Comparison of Compton-thick estimates with previous works using BAT spectral templates derived from the samples based on $N_{\mathrm{H}}$.

\begin{tabular}{lccccccc}
\hline \hline Paper & XLF $^{a}$ & $N_{\mathrm{H}}{ }^{b}$ & Templates & CTKR $^{c}$ & Datasets $^{d}$ & Flux $^{e}$ & $\chi_{\text {red }}^{2}$ \\
\hline G07 & H05 & G07 & G07 & $24 \div 26$ & ASCA SIS, HEAO1 & 10 & \\
& & & BAT & & & $<0.3$ & $>2$ \\
\hline T09 & U03 & T09 & $\begin{array}{c}\text { T09 } \\
\text { BAT }\end{array}$ & $24 \div 25$ & XMM, Chandra, BAT, INTEGRAL & 4 & \\
& & & & & $<1$ & $>2$ \\
\hline U14 & \multirow{2}{*}{ U14 } & U14 & U14 & $24 \div 26$ & ASCA SIS, BAT, INTEGRAL & $9 \pm 4$ & \\
& & & BAT & & & $9.5 \pm 0.5$ & 1.74 \\
\hline
\end{tabular}

Notes. For each paper we report both the CTK flux estimated in the original paper and the one estimated with the BAT templates, togheter with the $\chi_{\text {red }}^{2}$ of the fit. ${ }^{(a)}$ XLF used in the corresponding paper. ${ }^{(b)} N_{\mathrm{H}}$ distribution used in the corresponding paper. ${ }^{(c)} \log \left(N_{\mathrm{H}}\right)$ range of CTK AGN considered in the synthesis. ${ }^{(d)}$ Datasets used to compare with the CXB synthesis. ${ }^{(e)}$ Flux at the peak of the CTK component in the synthesis, in unit of $\mathrm{keV} \mathrm{cm}^{-2} \mathrm{~s}^{-1} \mathrm{Str}^{-1}$.

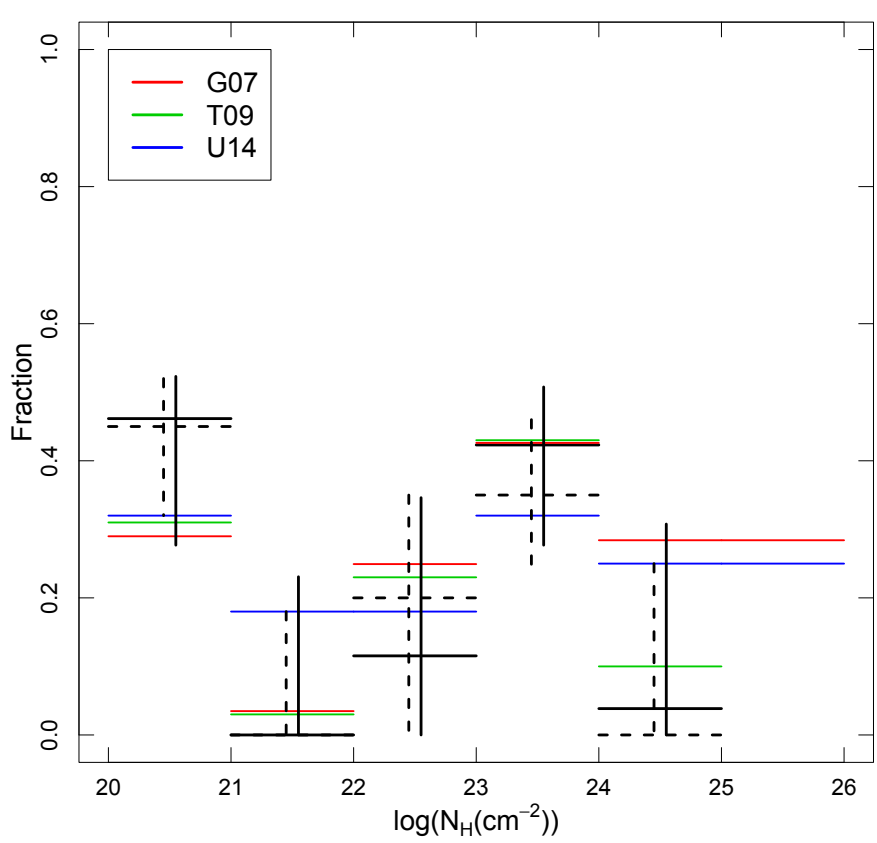

Fig. 7. Best fit $N_{\mathrm{H}}$ distribution with U14 XLF (continuous black lines) and U03 (dashed black lines) compared with $N_{\mathrm{H}}$ distribution from previous works. Error bars show the $1 \sigma$ confidence region. To compare better with previous works, here the $N_{\mathrm{H}}$ distributions are renormalised up to $N_{\mathrm{H}}=10^{24} \mathrm{~cm}^{-2}$.

threshold in order to estimate the AGN fraction which fails to be detected. We estimated that $11 \%$ of the mildly obscured AGN at redshift $<1.3$ have too faint spectra to be detected even in deep Chandra observations, adding an additional source of uncertainty to the XLF normalisation.

To account for these uncertainties in the fitting process, we introduced a scaling factor, which allows for a renormalisation of the synthesised spectrum on the data. The resulting factors vary from 1 to 1.3 for different XLFs.

Figure 7 shows the best fit $N_{\mathrm{H}}$ distributions using the XLF of U14 (continuous black lines) and U03 (dashed black lines), compared with the $N_{\mathrm{H}}$ distributions of G07, T09 and U14. The $N_{\mathrm{H}}$ distribution fit uncertainties are such that they are compatible with the $N_{\mathrm{H}}$ distributions published previously.

Table 5 shows the CTK fraction estimated for several XLFs and CTK bin sizes. Figure 8 shows the CXB synthesis obtained with the $N_{\mathrm{H}}$ distribution (best fit and $1 \sigma$ upper limit for the CTK fraction) that corresponds to the first row of Table 5. All the above combinations are able to represent the data, providing
Table 4. Compton-thick estimates obtained using BAT spectral templates derived from the samples based on absorption, fitting only the CTK density, for various combination of XLFs and $N_{\mathrm{H}}$ distributions.

\begin{tabular}{ccccc}
\hline \hline XLF & $N_{\mathrm{H}}$ & Flux $^{a}$ & $f_{\text {CTK }}{ }^{b}$ & $\chi_{\text {red }}^{2}$ \\
\hline H05 & G07 & $3.0 \pm 0.5$ & $24 \%$ & 2.12 \\
U03 & T09 & $0_{-0}^{+1.0}$ & $<5 \%$ & 1.92 \\
U14 & U14 & $11.5 \pm 0.5$ & $47 \%$ & 1.92 \\
M15 & $\mathrm{U} 14$ & $8.0 \pm 0.5$ & $47 \%$ & 1.64 \\
\hline
\end{tabular}

Notes. The datasets used in the fitting process are ROSAT, Swift/XRT, Swift/BAT, and INTEGRAL. ${ }^{(a)}$ Flux at the peak of the CTK component in the synthesis, in unit of $\mathrm{keV} \mathrm{cm}^{-2} \mathrm{~s}^{-1} \mathrm{Str}^{-1}$. ${ }^{(b)}$ Fraction of CTK $\left(\log \left(N_{\mathrm{H}}\right)>24\right)$ over all AGN (any $N_{\mathrm{H}}$, CTK themselves included).

Table 5. Compton-thick estimates obtained by fitting the whole $N_{\mathrm{H}}$ distribution.

\begin{tabular}{ccccccc}
\hline \hline XLF & CTKR & Templates & SF $^{a}$ & Flux $^{b}$ & $f_{\text {CTK }}$ & $\chi_{\text {red }}^{2}$ \\
\hline U14 & $24 \div 25$ & BAT $N_{\mathrm{H}}$ & 1.3 & $0.4_{-0}^{+2.9}$ & $<25 \%$ & 0.99 \\
$\mathrm{U} 14$ & $24 \div 25$ & BAT R11 & 1.3 & $0_{-0}^{+3}$ & $<22 \%$ & 1.10 \\
$\mathrm{U} 14$ & $24 \div 26$ & BAT $N_{\mathrm{H}}$ & 1.3 & $0.5_{-0}^{+2.9}$ & $<36 \%$ & 0.99 \\
$\mathrm{U} 03$ & $24 \div 25$ & BAT $N_{\mathrm{H}}$ & 1 & $0_{-0}^{+2.1}$ & $<12 \%$ & 1.17 \\
\hline
\end{tabular}

Notes. The datasets used for the fit are ROSAT, Swift/XRT, Swift/BAT, and INTEGRAL. We tested different combination of XLFs, $\log \left(N_{\mathrm{H}}\right)$ range of the CTK AGN, and templates (based on spectra stacked according to $N_{\mathrm{H}}$ or R11 definition. We report the scaling factor found, Flux of the CTK component, CTK fraction and $\chi_{\text {red }}^{2}$. (a) Scaling factor. ${ }^{(b)}$ Flux at the peak of the CTK component in the synthesis, in unit of $\mathrm{keV} \mathrm{cm}^{-2} \mathrm{~s}^{-1} \mathrm{Str}^{-1}$.

acceptable $\chi_{\text {red }}^{2}$. Table 5 shows that the scaling factor is always 1.3 with U14 and 1 with U03 XLF. We consider that such scaling factors are still acceptable, considering the uncertainties in the data, XLF parameters and the unresolved sources.

\section{Discussion}

\subsection{Stacked BAT spectra}

The unified model assumes that different classes of AGN can be explained by the observer line of sight crossing an anisotropic absorbing medium or not doing so (Antonucci 1993; Urry \& Padovani 1995). This does not explain why MOB sources feature 

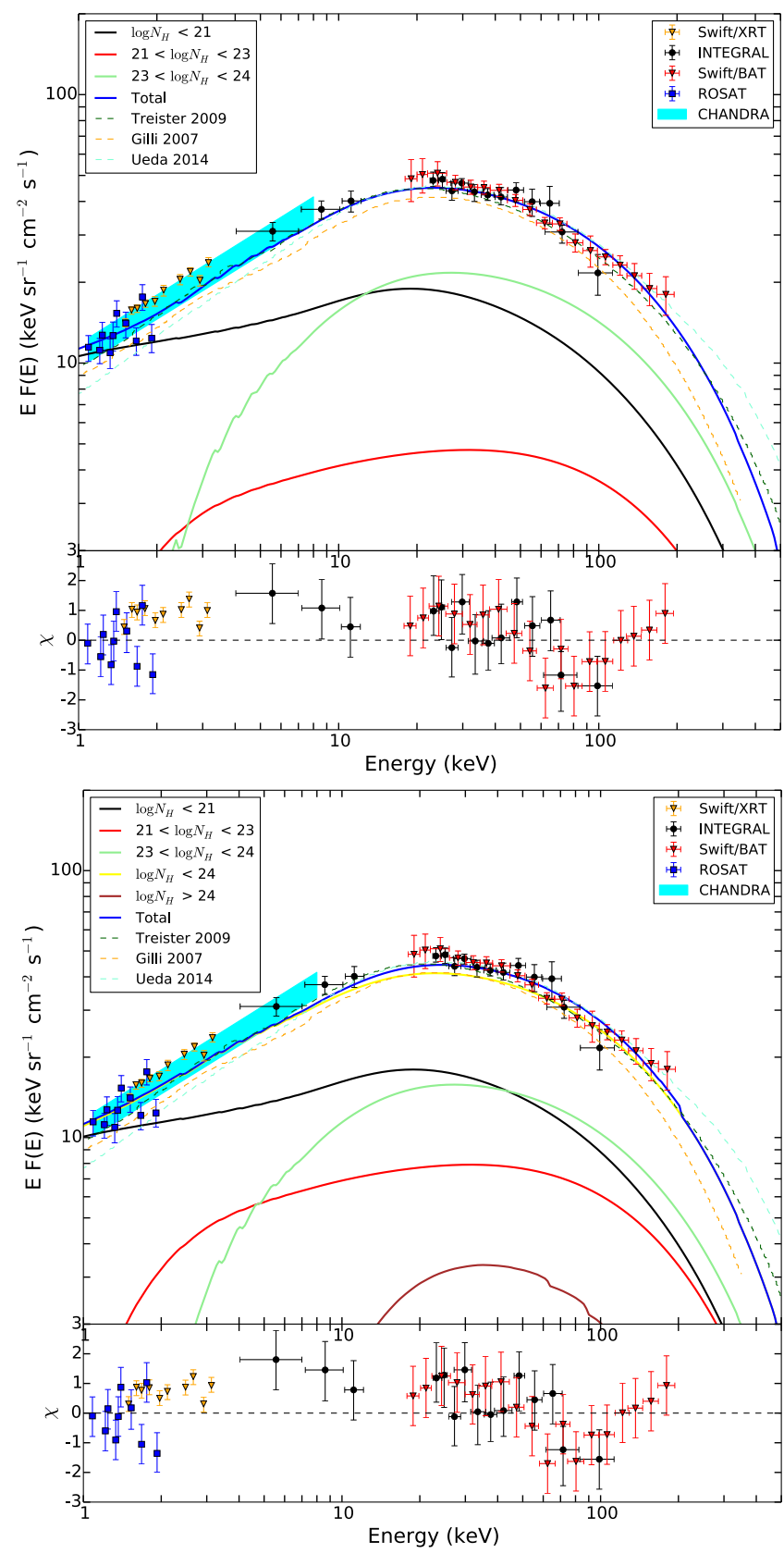

Fig. 8. Top panel: synthesis of the CXB spectrum and residuals (in unit of $\chi^{2}$ ) obtained using the best fit $N_{\mathrm{H}}$ distribution derived with the selection of Table 5, row 1. Bottom panel: as in the top panel, but using the $N_{\mathrm{H}}$ distribution corresponding to the $1 \sigma$ upper limit of the CTK fraction. Previously published CXB synthesis from T09, G07, and U14 (dotted lines) are also shown.

increased reflection. A clumpy absorber model can offer a solution, because obscuration and reflection could increase with the number of clumps. This geometry was put forward independently to explain high resolution infrared spectra of AGN (Ramos Almeida et al. 2011; Elitzur 2012).

The BAT spectra of Compton-thin AGN are modelled with the pexrav model with a cutoff energy fixed at $200 \mathrm{keV}$. There is a degeneracy between the cutoff energy and the reflection. Figure 9 shows the confidence contours as a function of $E_{\mathrm{c}}$ and $R$ for the spectra of the LOB 1 and MOB samples, respectively, and indicates that the stronger reflection observed in MOB sources is solid and not an effect of the fixed cutoff energy.

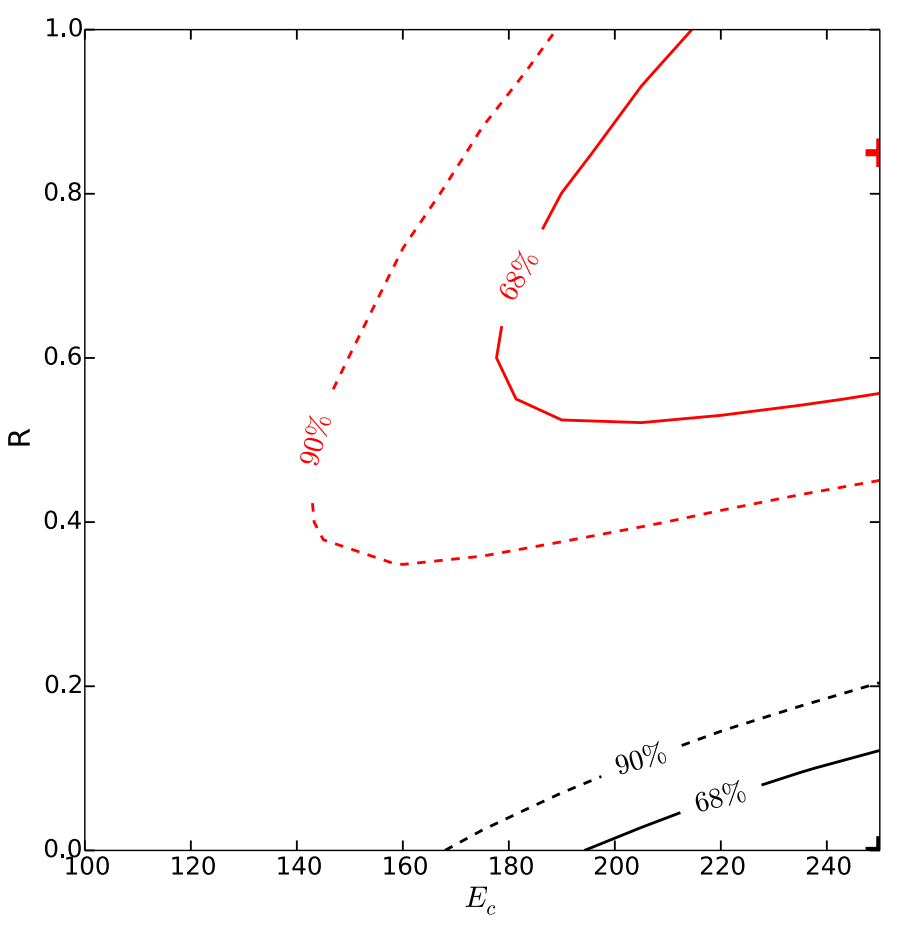

Fig. 9. Contour plot of the $\chi^{2}$ as a function of $E_{\mathrm{c}}$ and $R$ for the LOB1 $\left(10^{21}<N_{\mathrm{H}}<10^{22} \mathrm{~cm}^{-2}\right)$ spectrum (black) and the MOB $\left(10^{23}<N_{\mathrm{H}}<\right.$ $10^{24} \mathrm{~cm}^{-2}$ ) spectrum (red). Continuous lines show the $1 \sigma(68 \%)$ confidence region, dotted lines show the $90 \%$ confidence region. The crosses mark the best fit position.

To verify the effect of the fixed cutoff energy on the CXB synthesis, we also derived the best fit parameters for $E_{\mathrm{c}}=$ $150 \mathrm{keV}$ and $E_{\mathrm{c}}=250 \mathrm{keV}$ and performed the synthesis with these templates. The bump at $\sim 30 \mathrm{keV}$ is reproduced well in all cases, and the templates contribute to the synthesis in the same way, because the spectral models remain a good representation of the data. Changing the cutoff energy does not significantly change the spectral index, and therefore the flux of the template in the energy band of the XLF (2-10 keV for U14) is not significantly different for these cases, leading to similar normalisations in the CXB synthesis.

As mentioned in Sect. 4, previous works (e.g. G07; U14) introduced a Gaussian dispersion on the spectral index of their templates to reproduce the variance of the observed spectral slopes. The effect of this on the synthesis is to increase the flux of the CXB at hard X-rays (G07). We checked the effect of adding a spectral index dispersion in our synthesis and noticed that in our case the effect is not as strong as the BAT spectra already measure the spectral shape in the range 15 to $150 \mathrm{keV}$. We therefore did not introduce a spectral index distribution, also because the BAT templates already average the contribution of many sources.

\subsection{Contribution of Compton thick AGN}

Figure 10 shows the contour plot of the fraction of MOB sources and of the flux at the peak of the CTK contribution for the various models listed in Table 5. The scaling factor added in the fitting procedure is taken into account. All the contour plots show the expected tendency that the fraction of MOB decreases in order to allow for a stronger CTK contribution. For all the combinations investigated, the best fit indicates a CTK flux at the peak less than $1 \mathrm{keV} \mathrm{cm}^{-2} \mathrm{~s}^{-1} \mathrm{Str}^{-1}$ and a $1 \sigma$ upper limit of $2.1-2.9 \mathrm{keV} \mathrm{cm}^{-2} \mathrm{~s}^{-1} \mathrm{Str}^{-1}$ for the U03 and U14 XLFs 


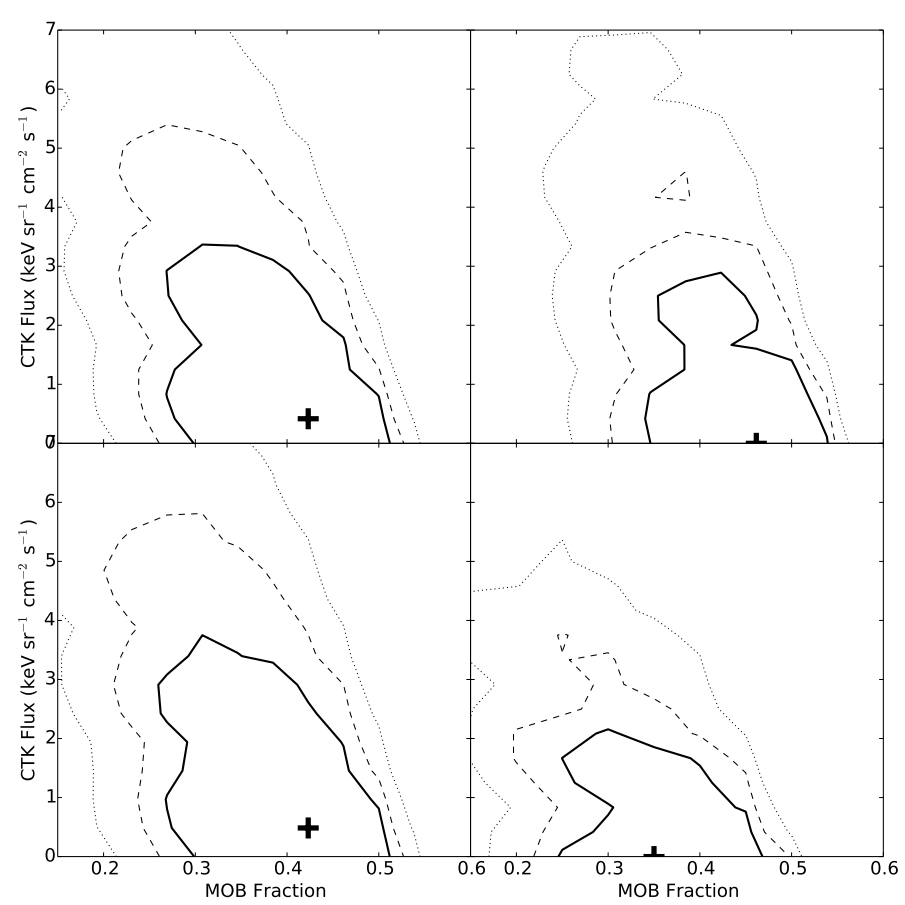

Fig. 10. Contour plot of MOB fraction versus the Flux of the CTK contribution in the $\mathrm{CXB}$ at $30 \mathrm{keV}$ (peak of the integrated spectrum) for the different assumptions used in Table 5: top left: row 1, top right: row 2, bottom left: row 3, bottom right: row 4 . Crosses indicate the best fit position. Continuous, dashed, and dotted lines represent the $68 \%(1 \sigma)$, $90 \%$ and $99 \%$ confidence regions respectively.

respectively, corresponding to $4-6 \%$ of the $\mathrm{CXB}$ emission. These fluxes correspond to a CTK emission that is two (T09) to three times (U14 and G07) fainter than that estimated in previous works.

Our fluxes correspond to a maximum percentage of CTK AGN between $12 \%$ (for U03 XLF) and 21\% (for U14 XLF), assuming that all CTK objects have $N_{\mathrm{H}}<10^{25} \mathrm{~cm}^{-2}$. Since CTK objects are mostly undetected, it is not clear where the $N_{\mathrm{H}}$ distribution should end. In the CXB modelling, the maximum absorption of CTK sources is typically considered to be $N_{\mathrm{H}}=10^{25} \mathrm{~cm}^{-2}$ (e.g. T09) or $N_{\mathrm{H}}=10^{26} \mathrm{~cm}^{-2}$ (e.g. G07; U14). The CTK fraction thus depends on the $N_{\mathrm{H}}$ distribution of the CTK sources, which is unknown: previous works simply assumed it to be constant. Adding a separate spectral template for deeply obscured CTK sources $\left(25<\log \left(N_{\mathrm{H}}\right)<26\right)$ in our fitting procedure, we found that their fraction is basically unconstrained by the data. Fixing it to the same value as of mildly obscured CTK sources we get a CTK fraction between $21 \%$ (for U03 XLF) and 29\% (for U14 XLF).

In the fitting procedure of the $N_{\mathrm{H}}$ distribution (Sect. 6.3) we added a scaling factor in order to account for various systematics effect. We did not introduce this scaling factor when fitting the Compton thick contribution alone (Sect. 6.2) because there we wanted to use exactly the same hypotheses as used in previous works, in order to verify the effect of the BAT templates on the CTK fraction estimation. We found that using these assumptions and the measured hard X-ray templates, does not provide a good fit to the CXB.

In the Swift/BAT catalogue of local AGN, Burlon et al. (2011) found that only $5.5 \%$ of them are classified as CTK $\left(\log \left(N_{\mathrm{H}}\right)>24\right)$, while Ricci et al. (2015) using a larger sample of Swift/BAT sources, found a value of $7 \%$. In the Chandra south deep field this percentage goes up to $20 \%$ (Brightman \& Ueda 2012). The discrepancy between the result of Swift/BAT and Chandra is related to the different sensitivities of the two instruments: Burlon et al. (2011) estimated that correcting for the bias against detection of very absorbed sources the intrinsic CTK AGN fraction over the total AGN is $20 \%$, in agreement with Brightman \& Ueda (2012). As most of these CTK objects have $N_{\mathrm{H}}<10^{25} \mathrm{~cm}^{-2}$ this is consistent with our upper limit of $21 \%$. We therefore conclude that a population of Compton-thick AGN larger than that effectively observed is not required to account for the CXB.

The CXB spectrum is dominated by low luminosity sources, but also by low redshift sources: $99 \%$ of the CXB flux is generated by AGN located at $z<1.3$. As a consequence, the CXB cannot be used to constrain galaxy evolution at high redshift. It is as yet unclear if mergers of galaxies in the early Universe have triggered rapid black-hole growth, stellar formation, and obscuration (Hopkins et al. 2006), or if the evolution has been more linear (Caplar et al. 2015). The stronger reflection observed in mildly obscured sources locally should be probed at higher redshift to study how the quasar environment evolved (Alexander et al. 2013).

\section{Summary and conclusions}

We measured the averaged hard X-ray spectral properties of several samples of Seyfert galaxies by stacking BAT spectra. We found that mildly obscured sources $\left(10^{23}<N_{\mathrm{H}}<10^{24} \mathrm{~cm}^{-2}\right)$ feature a stronger reflection than less absorbed sources, suggesting that AGN are surrounded by a clumpy rather than by a donut shaped torus.

The stacked BAT spectra have been used to define spectral templates of Seyfert galaxies and to synthetise the diffuse cosmic X-ray background. We found that the strong reflection of mildly obscured sources contribute significantly to the bulk of the CXB emission, leaving little space for the contribution of Compton-thick sources, in contrast with the results of previous works. The percentage of Compton thick sources estimated from our synthesis is less than $21 \%$, compatible with what is obtained from deep surveys.

We investigated possible systematic effects in the synthesis process attributable to assumptions in the modelling, using different XLF and spectral templates built on different samples. We also considered the effect of including or excluding the contribution of strongly obscured Compton thick sources $\left(10^{25}<N_{\mathrm{H}}<\right.$ $10^{26} \mathrm{~cm}^{-2}$ ). In all cases, only $4-6 \%$ of the CXB flux at $30 \mathrm{keV}$ can be attributed to Compton-thick sources.

We allowed a renormalisation factor on the absolute flux of the CXB synthesis to account for the uncertainties in the CXB measurements and in the XLF parameters. The Monitor Vsego Neba (MVN) X-ray astronomical experiment (Revnivtsev et al. 2012) will help to improve our knowledge of the CXB normalisation.

\section{References}

Aird, J., Nandra, K., Laird, E. S., et al. 2010, MNRAS, 401, 2531

Aird, J., Coil, A. L., Georgakakis, A., et al. 2015, MNRAS, 451, 1892

Ajello, M., Greiner, J., Sato, G., et al. 2008, ApJ, 689, 666

Akylas, A., Georgakakis, A., Georgantopoulos, I., Brightman, M., \& Nandra, K. 2012, A\&A, 546, A98

Alexander, D. M., Bauer, F. E., Brandt, W. N., et al. 2003, AJ, 126, 539

Alexander, D. M., Stern, D., Del Moro, A., et al. 2013, ApJ, 773, 125

Antonucci, R. 1993, ARA\&A, 31, 473 
V. Esposito and R. Walter: Mildly obscured active galaxies and the cosmic X-ray background

Barthelmy, S. D., Barbier, L. M., Cummings, J. R., et al. 2005, Space Sci. Rev., 120,143

Baumgartner, W. H., Tueller, J., Markwardt, C. B., et al. 2013, ApJS, 207, 19

Brightman, M., \& Nandra, K. 2011, MNRAS, 413, 1206

Brightman, M., \& Ueda, Y. 2012, MNRAS, 423, 702

Brightman, M., Nandra, K., Salvato, M., et al. 2014, MNRAS, 443, 1999

Buchner, J., Georgakakis, A., Nandra, K., et al. 2015, ApJ, 802, 89

Burlon, D., Ajello, M., Greiner, J., et al. 2011, ApJ, 728, 58

Caplar, N., Lilly, S. J., \& Trakhtenbrot, B. 2015, ApJ, 811, 148

Churazov, E., Sunyaev, R., Revnivtsev, M., et al. 2007, A\&A, 467, 529

Comastri, A., Setti, G., Zamorani, G., \& Hasinger, G. 1995, A\&A, 296, 1

De Luca, A., \& Molendi, S. 2004, A\&A, 419, 837

Elitzur, M. 2012, ApJ, 747, L33

Frontera, F., Orlandini, M., Landi, R., et al. 2007, ApJ, 666, 86

Gehrels, N., Chincarini, G., Giommi, P., et al. 2004, ApJ, 611, 1005

Gendreau, K. C., Mushotzky, R., Fabian, A. C., et al. 1995, PASJ, 47, L5

Giacconi, R., Gursky, H., Paolini, F. R., \& Rossi, B. B. 1962, Phys. Rev. Lett., 9, 439

Giacconi, R., Zirm, A., Wang, J., et al. 2002, ApJS, 139, 369

Gilli, R., Comastri, A., \& Hasinger, G. 2007, A\&A, 463, 79 (G07)

Gruber, D. E., Matteson, J. L., Peterson, L. E., \& Jung, G. V. 1999, ApJ, 520, 124

Hasinger, G. 2008, A\&A, 490, 905

Hasinger, G., Altieri, B., Arnaud, M., et al. 2001, A\&A, 365, L45

Hasinger, G., Miyaji, T., \& Schmidt, M. 2005, A\&A, 441, 417 (H05)

Hickox, R. C., \& Markevitch, M. 2006, ApJ, 645, 95

Hopkins, P. F., Hernquist, L., Cox, T. J., et al. 2006, ApJS, 163, 1

Jourdain, E., Götz, D., Westergaard, N. J., Natalucci, L., \& Roques, J. P. 2008, in Proc. of the 7th INTEGRAL Workshop, 144

Krimm, H. A., Holland, S. T., Corbet, R. H. D., et al. 2013, ApJS, 209, 14

La Franca, F., Fiore, F., Comastri, A., et al. 2005, ApJ, 635, 864
Magdziarz, P., \& Zdziarski, A. A. 1995, MNRAS, 273, 837

Malizia, A., Molina, M., Bassani, L., et al. 2014, ApJ, 782, L25

Marshall, F. E., Boldt, E. A., Holt, S. S., et al. 1980, ApJ, 235, 4

Miyaji, T., Ishisaki, Y., Ogasaka, Y., et al. 1998, A\&A, 334, L13

Miyaji, T., Hasinger, G., Salvato, M., et al. 2015, ApJ, 804, 104 (M15)

Moretti, A., Pagani, C., Cusumano, G., et al. 2009, A\&A, 493, 501

Ramos Almeida, C., Levenson, N. A., Alonso-Herrero, A., et al. 2011, ApJ, 731, 92

Ranalli, P., Koulouridis, E., Georgantopoulos, I., et al. 2016, A\&A, in press, DOI: $10.1051 / 0004-6361 / 201527013$

Revnivtsev, M., Gilfanov, M., Sunyaev, R., Jahoda, K., \& Markwardt, C. 2003, A\&A, 411, 329

Revnivtsev, M., Gilfanov, M., Jahoda, K., \& Sunyaev, R. 2005, A\&A, 444, 381

Revnivtsev, M., Semena, N., Akimov, V., et al. 2012, in SPIE Conf. Ser., 8443, 10

Ricci, C., Walter, R., Courvoisier, T. J.-L., \& Paltani, S. 2011, A\&A, 532, A102 (R11)

Ricci, C., Ueda, Y., Koss, M. J., et al. 2015, ApJ, 815, L13

Setti, G., \& Woltjer, L. 1989, A\&A, 224, L21

Treister, E., Urry, C. M., \& Virani, S. 2009, ApJ, 696, 110 (T09)

Tueller, J., Mushotzky, R. F., Barthelmy, S., et al. 2008, ApJ, 681, 113

Tueller, J., Baumgartner, W. H., Markwardt, C. B., et al. 2010, ApJS, 186, 378

Türler, M., Chernyakova, M., Courvoisier, T. J.-L., et al. 2010, A\&A, 512, A49

Ueda, Y., Akiyama, M., Ohta, K., \& Miyaji, T. 2003, ApJ, 598, 886 (U03)

Ueda, Y., Akiyama, M., Hasinger, G., Miyaji, T., \& Watson, M. G. 2014, ApJ, 786, 104 (U14)

Urry, C. M., \& Padovani, P. 1995, PASP, 107, 803

Vasudevan, R. V., Mushotzky, R. F., \& Gandhi, P. 2013, ApJ, 770, L37

Walter, R., \& Cabral, N. 2009, A\&A, 497, 97

Worsley, M. A., Fabian, A. C., Bauer, F. E., et al. 2005, MNRAS, 357, 1281

Yaqoob, T. 2012, MNRAS, 423, 3360 